\title{
Epidemiology and outcome of Staphylococcus aureus bloodstream infection and sepsis in a Norwegian county 1996-2011: an observational study
}

Julie Paulsen ${ }^{1,2,8^{*}}$, Arne Mehl ${ }^{1,2}$, Åsa Askim ${ }^{3,4}$, Erik Solligård ${ }^{3,4}$, Bjørn Olav Åsvold ${ }^{5,7}$ and Jan Kristian Damås 1,6

\begin{abstract}
Background: Staphylococcus aureus is one of the most common and lethal causes of bloodstream infection and the incidence is increasing. We carried out a prospective observational study of patients with Staphylococcus aureus bloodstream infection and sepsis in Nord-Trøndelag county in Norway from 1996-2011. The main outcome of interest was all-cause mortality within 30 and 90 days.
\end{abstract}

Methods: Positive blood cultures were registered prospectively by the microbiology laboratory and clinical variables were retrospectively registered from patients' hospital records. The severity of sepsis was assigned according to the 2001 International Sepsis Definition Conference criteria. The association between clinical characteristics and mortality was studied using logistic regression analysis, and adjusted 30- and 90-day mortality risks were estimated.

Results: Among 373 patients, the median age was 74 years and $60.3 \%$ were male. $0.8 \%$ of the patients were diagnosed with MRSA. 29.8\% of the patients developed severe sepsis and 12.9\% developed septic shock. The all-cause mortality was $14.5 \%, 27.3 \%$ and $36.2 \%$ at 7, 30 and 90 days, respectively. Compared to patients with sepsis without organ failure (Mortality risk 13.3\%, 95\% Cl 7.5-16.3\%), the 30-day mortality risk was 3-fold higher among those with severe sepsis $(39.9 \%, 95 \% \mathrm{Cl}$ 29.5-48.5\%) and more than 4-fold higher for those with septic shock (57.3\%, 95\% Cl 42.5-72.2\%). The 30-day all-cause mortality varied by focus of infection, with the highest 30-day mortality risk among those with a pulmonary focus $(42.4 \%, 95 \% \mathrm{Cl} 26.0-58.5 \%)$ and unknown focus of infection (38.7\%, 95\% Cl 27.5-48.2\%). The mortality risk did not differ between the first and second halves of the study period with a 30-day mortality risk of $27.3 \%$, (95\% Cl 18.1-33.1\%) for $1996-2003$ versus $27.4 \%$ (95\% Cl 19.4-31.4\%) for 2004-2011. The same pattern was seen for 90-day mortality risk.

Conclusion: Staphylococcus aureus bloodstream infection carries a high case fatality rate, especially among those with severe sepsis and septic shock and among those with a pulmonary or unknown focus of infection. There was no decrease in 30- or 90-day mortality risk during the study period. This underscores the importance of continuing surveillance and efforts to improve the outcome of this serious disease.

Keywords: Staphylococcus aureus, Bacteremia, Sepsis, Organ failure, Comorbid disease, Focus of infection

\footnotetext{
* Correspondence: julie.paulsen@ntnu.no

${ }^{1}$ Centre of Molecular Inflammation Research, Department of Cancer Research and Molecular Medicine, Norwegian University of Science and Technology, Trondheim, Norway

2Department of Medicine, Levanger Hospital, Nord-Trøndelag Health Trust, Levanger, Norway

Full list of author information is available at the end of the article
}

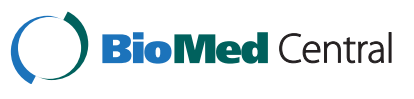

(C) 2015 Paulsen et al.; licensee BioMed Central. This is an Open Access article distributed under the terms of the Creative Commons Attribution License (http://creativecommons.org/licenses/by/4.0), which permits unrestricted use, distribution, and reproduction in any medium, provided the original work is properly credited. The Creative Commons Public Domain Dedication waiver (http://creativecommons.org/publicdomain/zero/1.0/) applies to the data made available in this article, unless otherwise stated. 


\section{Background}

Staphylococcus aureus is one of the most lethal and common causes of bloodstream infection, with an incidence of 26/100 000 population/year [1]. Of concern, a $34 \%$ increase in incidence has been observed in Europe from 2002-2009 [2]. Factors contributing to the role of Staphylococcus aureus as a public health problem include its affinity to foreign objects such as intravenous lines and prosthetic material, and its propensity to generate metastatic foci and complicated disease [3]. Another challenge is its ability to quickly develop resistance to antimicrobial agents [4]. Even in populations with a low level of antibiotic resistance, Staphylococcus aureus is a cause of severe bloodstream infection with high mortality [5]. Despite improvements in survival over the last three decades, the 30 day all-cause mortality rates are still at $17-39 \%[5-10]$.

Several studies have shown that the outcome of Staphylococcus aureus bloodstream infection may differ by focus of infection, with unidentified focus, respiratory focus and endocarditis being associated with the highest mortality [8]. In addition, an uneradicated or noneradicable focus has been associated with increased mortality $[11,12]$. Older age, increasing number and types of comorbid diseases before the onset of infection and clinical severity of the bloodstream infection have also been associated with reduced survival [13].

The clinical characteristics and outcome of Staphylococcus aureus bloodstream infection are well described in many Western countries [8,14]. However, it is important to study the clinical outcome of bloodstream infections in multiple populations and at multiple time points both to evaluate differences in disease characteristics between populations and to gauge the development over time. It is important to investigate the characteristics of the disease in order to identify areas where management can be improved. Internationally, there has been an increasing effort to improve the management and outcome of sepsis including bloodstream infection over the last decades with initiatives such as the international Surviving Sepsis Campaign $[15,16]$. In order to improve follow up and treatment of this patient group we carried out a prospective observational study of Staphylococcus aureus bloodstream infection in Nord-Trøndelag County.

\section{Methods}

\section{Setting and population}

Nord-Trøndelag is a county in Central Norway with a current population of 134864 . It is served by two community hospitals, Namsos Hospital and Levanger Hospital. The closest tertiary referral hospital is St Olavs University Hospital in Trondheim. We included all patients $\geq$ age 16 diagnosed with Staphylococcus aureus bloodstream infection at Levanger Hospital between 1996 and 2011, and at Namsos Hospital between 1999 and 2011. For residents of NordTrøndelag who in 1995-97 had participated in a population survey (the HUNT2 survey) we could also include infections detected at St. Olavs Hospital between 1996 and 2011. All adults in Nord-Trøndelag were invited to the survey and $69.5 \%$ participated [17]. All positive blood cultures have been prospectively registered by the clinical microbiology laboratory in Levanger, Namsos and at St. Olavs Hospital. BACTEC 9240 (Becton Dickinson Diagnostic Instrument Systems, Sparks, MD) was used for blood culture testing [18]. Resistance testing was performed by disc diffusion. Methicillin resistance was tested with a cefoxitin disc. Oxacillin-resistant isolates were sent to St Olavs Hospital for testing of the mecA gene.

\section{Patient characteristics}

Clinical information was gathered retrospectively from the patients' hospital records. All data were collected using a standardized data retrieval form assessing patient characteristics, comorbid conditions, results of investigations and treatment. The data collection was carried out by trained research nurses and all registered data was secondarily assessed either by an infectious disease consultant or the first author of this study. An episode of bloodstream infection was defined as the presence of one or more microorganism(s) in blood culture along with clinical evidence of infection. If a patient had more than one episode of Staphylococcus aureus bacteremia during the study period, only the first was included. We decided to include patients with polymicrobial infection in this study since a bloodstream infection containing Staphylococcus aureus should be regarded as clinically significant [19].

The setting of infection was classified as hospitalacquired (HA), healthcare-associated (HCA) or community-acquired (CA) as defined by Friedman et al. [20], with the exception that only patients that were hospitalized for two or more days in the 30, as opposed to 90 days prior to the infection were classified as having a HCA infection, in keeping with the definition used by Shorr et al. [21]. The number and severity of combined comorbid conditions were assessed according to the Charlson weighted Comorbidity Index (CCI) [22]. Mortality was measured as all-cause mortality within day 7, 30 and 90. By using the 11digit unique identification number of all Norwegian citizens, electronic hospital records in Norway are updated with mortality data from the Norwegian population registry so that mortality data after discharge from hospital can be reliably assessed. 


\section{Severity of disease}

We graded the severity of disease (sepsis, severe sepsis and septic shock) according to the 2001 International Sepsis Conference definition [15]. In our cohort we defined sepsis as documented bloodstream infection and two or more of the following: temperature $\geq 38.3^{\circ} \mathrm{C}$ or $<36.0^{\circ} \mathrm{C}$, heart rate $>90$ beats/minute, respiratory rate $>20 / \mathrm{min}$ or $\mathrm{PaCO}_{2}<4.3 \mathrm{kPa}$ or mechanical ventilation due to acute respiratory failure, glucose $>7.7 \mathrm{mmol} / \mathrm{l}$ in the absence of diabetes, leucocytes $>12 \times 10^{9} / 1$ or $<4 \times 10^{9} / \mathrm{l}$, elevated CRP or procalcitonin, acute hypotension (systolic BT $<90 \mathrm{mmHg}$, MAP $<70 \mathrm{mmHg}$ or a fall of $\geq 40 \mathrm{mmHg}$ ), or significant positive fluid balance ( $>20 \mathrm{ml} / \mathrm{kg}$ over 24 hours).

Severe sepsis was defined as sepsis and sepsis-related dysfunction in at least one organ, or hypoperfusion or hypotension. Acute organ dysfunction was defined as mental confusion, arterial hypoxemia, acute oliguria, increase in serum creatinine of $\geq 45 \mu \mathrm{mol} / \mathrm{l}$, low platelet count, coagulation disturbance, ileus, or hyperbilirubinemia. The specific criteria for organ dysfunction used were the same as those outlined in the 2001 International Sepsis Conference definition [15].

Septic shock was defined as sepsis and systolic blood pressure $<90 \mathrm{mmHg}$ or a fall in systolic blood pressure of at least $40 \mathrm{mmHg}$ despite at least $1000 \mathrm{ml}$ fluid resuscitation or vasopressor needed to maintain systolic blood pressure $>90 \mathrm{mmHg}$ and evidence of organ failure.

Any alteration in disease severity was registered, and the timing and extent of the most pronounced degree of organ dysfunction was noted and used to define whether the patient had sepsis, severe sepsis or septic shock. For those who did not show any evidence of deterioration during the episode, the day of positive blood culture was registered as the day with the most severe affection. Severity of disease was also assessed by the Pitt bacteremia score [23].

\section{Focus of infection}

Reported signs of infection along with focal growth of the same microbe as in blood culture was taken as a confirmation of skin, soft tissue, joint or surgical infection. Respiratory focus was diagnosed with clinical signs of respiratory infection accompanied by positive radiologic findings. Intravenous line infection was diagnosed with growth of Staphylococcus aureus from the tip of the catheter as well as in peripheral blood, and also if the microbe was isolated from pus around the catheter entry site. If clinical signs of catheter infections were present without local growth and no other focus was detected an intravenous line infection was registered as likely. Staphylococcus aureus has also been shown to be a likely ascending urinary pathogen among patients with urinary tract catheterization or manipulation [24,25]. A urinary focus was assigned when there was growth of bacteria in the urine as well as in blood along with clinical signs/ symptoms or risk factor for urinary infection, and no other source of infection was identified. Endocarditis was registered if diagnosed according to clinical and echocardiographic criteria during the hospital stay. An unknown focus of infection was assigned when none of the criteria for ascertaining a focus were met.

\section{Management}

Empiric antibiotic treatment was defined as treatment given before the pathogen was known. Definitive treatment was defined as treatment administered after result of the blood cultures was available. Inefficient antibiotic treatment was defined as treatment to which the microbe was resistant. Treatment was defined as efficient as long as the microbe was sensitive, even if the drug administered was not the first choice.

\section{Ethical considerations}

The study was approved by the Central Norway Regional Committee for Medical Health and Research Ethics. Since no patient contact or intervention was carried out, the need for informed consent was waived.

\section{Statistical analysis}

Data was analyzed using SPSS for Windows (Version 21, Armonk, NY: IBM Corp) and STATA version 13 (StataCorp LP, College Station, Texas). The associations between clinical characteristics and 30- and 90-day mortality were investigated using logistic regression analysis where odds ratios (ORs) with 95\% confidence intervals (CIs) were estimated. In addition, we studied the association between clinical characteristics and severe sepsis/septic shock as an outcome, in order to identify groups of patients particularly vulnerable to develop severe disease and need a higher level of follow-up and care. All associations were estimated both unadjusted and adjusted for potential confounders. Thus, all analyses were adjusted for sex and age group $(<60,60-69,70-79$ and $\geq 80$ years). For place of acquisition, focus, severity and time period, the associations were additionally adjusted for prior comorbid conditions using three categories of the CCI $(0,1-2$ and $\geq 3)$. For the association between individual comorbid conditions and 30- and 90day mortality, those not having the condition in question were used as reference. Adjusted 30- and 90-day mortality risks and risk of severe sepsis/shock were estimated from the logistic regression model. For ordinal variables, we tested for linear trend across categories by using the categories as a continuous variable in the logistic regression analysis. 


\section{Results}

We identified 402 episodes of Staphylococcus aureus bloodstream infection during the study period, of which 23 were recurrent episodes. Six patients did not meet the clinical criteria for sepsis, either because of a mild clinical course with only one elevated inflammatory parameter or because clinical or laboratory data needed to assess whether these patients met the sepsis criteria were lacking. For this reason we chose to exclude these patients thus leaving 373 patients for further analysis. 25 patients $(6.7 \%)$ had polymicrobial infection. 3 patients $(0.8 \%)$ were diagnosed with MRSA, 2 of these were in the setting of a polymicrobial infection and all three episodes were healthcare-associated. Data on antibiotic susceptibility were available for the 348 monomicrobial isolates. $26.7 \%$ were penicillin-sensitive, $99.4 \%$ were dicloxacillin-sensitive, 98.5\% were gentamicin-sensitive (Table 1).

The median patient age was 74 years, $60.3 \%$ of the cases were male. Overall, $41.8 \%$ acquired the infection in a healthcare-associated setting (HCA), 29.2\% were hospital-acquired (HA) and $29.0 \%$ were communityacquired (CA). Previously known comorbid illness was present in $94.6 \%$ of the patients (Table 1). The all-cause mortality rate was $14.5 \%, 27.3 \%$ and $36.2 \%$ at 7,30 and 90 days, respectively (Table 2).

The 30-day mortality risk differed by age and by number and severity of comorbid conditions prior to the onset of infection. Compared to those having CCI score of 0 (30-day mortality risk $13.7 \%, 95 \%$ CI $7.0-21.8 \%$ ), the 30-day mortality risk was 2-fold higher among those with CCI score 1-2 (24.8\%, 95\% CI 17.6-31.0\%) and 3fold higher for those with CCI score of 3 or more (39.1\%, 95\% CI 30.0-47.6\%) (Table 3). The number and severity of comorbid conditions were similarly associated with 90-day mortality risk (Additional file 1: Table S1).

$57.4 \%$ of the patients had sepsis with no organ failure, $29.8 \%$ had severe sepsis and $12.9 \%$ had septic shock (Table 2). Compared to those with sepsis without organ failure (30-day mortality risk $13.3 \%$, 95\% CI 7.5-16.3\%), the 30-day mortality risk was 3-fold higher among those with severe sepsis $(39.9 \%, 95 \%$ CI 29.5-48.5\%) and more than 4-fold higher for those with septic shock (57.3\%, 95\% CI 42.5-72.2\%) (Table 4). A similar association was seen with 90-day mortality risk (Additional file 1: Table S2). A steady increase in mortality risk according to severity was also observed for the Pitt bacteremia score.

The focus of infection was ascertained in $74.5 \%$ of the patients, whereas in $25.5 \%$, no focus was identified. The most common focus was skin and soft tissue infections (19.6\%), whereas $4.8 \%$ were diagnosed with endocarditis. Overall, $35.9 \%$ of the patients were assessed with echocardiography (Table 2). Among those with no ascertained focus of infection, $29.8 \%$ were examined with echocardiography. The mortality varied by focus of infection. The highest 30-day mortality risk was seen among those with a respiratory focus $(42.4 \%, 95 \% \mathrm{CI} 26.0-58.5 \%)$ and unknown focus of infection (38.7\%, 95\% CI 27.5-48.2\%) (Table 4). The highest 90-day mortality risk was seen for the same foci (Additional file 1: Table S2). The lowest 30day risk was seen for urinary tract focus $(11.2 \%, 95 \% \mathrm{CI}$ 3.4-24.0\%), abscess (15.6\%, 96\% CI 4.3-35.4\%), and osteomyelitis/septic arthritis (13.8\%, 95\% CI 4.9-26.1\%) (Table 4). For these foci there was also a low risk at 90 days (Additional file 1: Table S2). The mortality risk for patients with endocarditis was low at 30 days (13.4\%, 95\% CI 2.8-37.4\%) (Table 4), but the risk increased nearly three-fold to $39.3 \%$ at 90 days (95\% CI 17.0 63.7\%) (Additional file 1: Table S2).

Overall, $24.7 \%$ of the patients were treated in the intensive care unit or a medical high dependency unit. Among those with septic shock $79.2 \%$ were admitted to the ICU or medical high dependency unit, whereas $30.6 \%$ of those with severe sepsis received care at the ICU or medical high dependency unit. The risk of acquiring severe sepsis/septic shock increased with age from $30.7 \%$ (95\% CI 21.7-41.6\%) for those aged $<60$ to $49.0 \%$ (95\%CI 40.3-57.7\%) for those aged $\geq 80$. Among individual comorbidities the risk of severe sepsis or septic shock was highest for those with heart failure (67.4\%, 95\% CI 51.2-80.6\%), renal failure $(60.5 \%$, 95\% CI $45.3-$ $73.9)$ and chronic pulmonary disease $(55.6 \%, 95 \% \mathrm{CI}$ 43.0-67.6\%). The infectious foci associated with the highest risk of developing severe disease included endocarditis $(86.0 \%, 95 \%$ CI $64.4-95.8 \%)$, respiratory focus $(52.4 \%$, 95\% CI 36.2-68.1\%) and unknown infectious foci (51.0\%, 95\% CI 40.6-61.3\%) (Additional file 1: Table S3).

The most common empirical antibiotic regimens administered were penicillin combined with an aminoglycoside (17.3\%), cefuroxime-containing regimens (16.9\%) and dicloxacillin-containing regimens (16.6\%). 2.9\% received no initial treatment. $15.3 \%$ received initial treatment that was not effective against the microbe in question, and most of these were treated with penicillin in monotherapy. As definitive treatment, $51 \%$ of the patients were treated with dicloxacillin-containing regimens. $2.7 \%$ received no definitive treatment and $2.9 \%$ received inefficient definitive treatment (Table 2). Among those with community-acquired infection, 31 patients $(28.7 \%)$ received inefficient or no treatment initially. The same was true for $16.7 \%$ of those with healthcare-acquired infection and $10.1 \%$ of those with hospital-acquired infection. 119 patients, (31.9\%) received surgical treatment including drainage of abscesses and pleural fluid, wound revisions, amputations, arthrocentesis, removal of orthopedic hardware, stenting of the renal pelvis or the common bile duct and cardiac valve surgery. The median duration of intravenous antibiotic therapy was 10 days and the meadian total duration 
Table 1 Patient and infection characteristics

\begin{tabular}{|c|c|}
\hline Characteristic & $\mathrm{N}(\%)$ \\
\hline Patients included & $373(100)$ \\
\hline \multicolumn{2}{|l|}{ Sex } \\
\hline Female & $148(39.7)$ \\
\hline Male & $225(60.3)$ \\
\hline \multicolumn{2}{|l|}{ Acquisition } \\
\hline Community-acquired & $108(29.0)$ \\
\hline Healthcare-associated & $156(41.8)$ \\
\hline Hospital-acquired & $109(29.2)$ \\
\hline \multicolumn{2}{|l|}{ Age category } \\
\hline$<60$ & $81(21.7)$ \\
\hline $60-69$ & $58(15.6)$ \\
\hline $70-79$ & $109(29.2)$ \\
\hline$\geq 80$ & $125(33.5)$ \\
\hline \multicolumn{2}{|l|}{ Comorbid conditions } \\
\hline No underlying illness & $20(5.4)$ \\
\hline Malignancy & $96(25.7)$ \\
\hline Renal failure & $45(12.1)$ \\
\hline Diabetes mellitus & $72(19.3)$ \\
\hline Hypertension & $114(30.6)$ \\
\hline Cardiovascular disease & $154(41.3)$ \\
\hline Heart failure & $40(10.7)$ \\
\hline Chronic pulmonary disease & $62(16.6)$ \\
\hline Rheumatic disease & $39(10.5)$ \\
\hline \multicolumn{2}{|l|}{ Charlson Comorbidity Index (CCI) } \\
\hline 0 & $85(22.8)$ \\
\hline $1-2$ & $160(42.9)$ \\
\hline$\geq 3$ & $128(34.3)$ \\
\hline \multicolumn{2}{|l|}{ Focus of infection } \\
\hline Unknown & $94(25.2)$ \\
\hline Respiratory focus & $37(9.9)$ \\
\hline Urinary tract & $33(8.8)$ \\
\hline Skin/soft tissue & $73(19.6)$ \\
\hline Abscess & $27(7.2)$ \\
\hline IV catheter & $28(7.5)$ \\
\hline Endocarditis & $18(4.8)$ \\
\hline Osteomyelitis/Septic arthritis & $44(11.8)$ \\
\hline Other* & $19(5.1)$ \\
\hline \multicolumn{2}{|l|}{ Microbiological characteristics } \\
\hline Polymicrobial infection & $25(6.7)$ \\
\hline Methicillin-resistant isolates & $3(0.8)$ \\
\hline Penicillin-sensitive isolates§ & $93(26.7)$ \\
\hline Dicloxacillin-sensitive isolates§ & $346(99.4)$ \\
\hline Gentamicin-sensitive isolates $\$ \$$ & $323(98.5)$ \\
\hline Gentamicin intermediate or resistant $\$ \$$ & $5(1.5)$ \\
\hline
\end{tabular}

Table 1 Patient and infection characteristics (Continued)

Time period

1996-2003

$144(38.6)$

2004-2011

229 (61.4)

${ }^{*}$ Other focus includes dental foci, parotitis, 2 cases of meningitis, mediastinits, an infected peritoneal dialysis catheter and one probable intravascular graft infection.

Sassessed for monomicrobial episodes, $\mathrm{n}=348$.

\$Data lacking for 20 isolates (5.7\%).

including oral antibiotic treatment was 14 days (Table 2). When examining the first (1996-2003) and second (2004-2011) halves of the study separately, $30.6 \%$ of the patients in the first period and $32.8 \%$ of the patients in the second period received surgical treatment. $32.6 \%$ of the patients in the first period were examined with echocardiography versus $38.0 \%$ in the second period of the study.

This study was carried out over a period of 16 years. The age, sex and comorbidity-adjusted 30-day mortality risk did not differ between the first and second halves of the study period with an adjusted mortality risk of $27.3 \%$ (95\% CI 18.1-33.1\%) for 1996-2003 versus 27.4\% (95\% CI 19.4-31.4\%) for 2004-2011 (Table 4). The same pattern was seen for 90-day adjusted mortality risk (Additional file 1: Table S2).

\section{Discussion}

This study highlights several important aspects regarding bloodstream infection and sepsis with Staphylococcus aureus. Only 6 patients out of 379 with positive blood cultures did not meet the criteria for sepsis, emphasizing the clinical relevance of a positive blood culture for Staphylococcus aureus. Our findings also confirm that the proportion of Staphylococcus aureus bloodstream infections caused by MRSA in Norway is low. There was a strong influence of age, comorbid burden, disease severity and focus of infection on the outcome, and there was a stable high case fatality rate of $27.3 \%$ during the study period. Our 30 day all-cause mortality rate is higher than in other Scandinavian studies $[7,26]$, but a clinical study of Staphylococcus aureus bacteremia in inpatients in Oxfordshire and one German study found similar mortality rates $[27,28]$. Some of these differences could be due to differences in study design and patient characteristics. One factor contributing to the high mortality rate in our population could be the old age of our patients. We also found a clear association between comorbid diseases and mortality, and a high proportion of our patients suffered from comorbid disease. The association between an increased CCI and mortality in patients with Staphylococcus aureus bacteremia has been shown earlier by Lesens et al. [29]. 
Table 2 Patient outcomes and management

\begin{tabular}{ll}
\hline Severity of sepsis & N (\%) \\
\hline Sepsis without organ failure & $214(57.4)$ \\
Severe sepsis & $111(29.8)$ \\
Septic shock & $48(12.9)$
\end{tabular}

Pitt bacteremia score

1

2

$\geq 3$

All-cause mortality

7-day

30-day

90-day

Empiric antibiotic management

Penicillin plus aminoglycoside

$65(17.3)$

Cefuroxime-containing regimens

$63(16.9)$

Dicloxacillin-containing regimens

$62(16.6)$

Penicillin monotherapy

$52(13.9)$

Cefotaxime-containing regimens

Other treatment

$47(12.7)$

$73(19.6)$

No treatment

11 (2.9)

$57(15.3)$

Definitive antibiotic treatment*

Penicillin plus aminoglycoside

$18(4.8)$

Dicloxacillin-containing regimens

$190(51.0)$

Penicillin monotherapy

$34(9.1)$

Cefuroxime-containing regimens

$29(7.9)$

Cefotaxime-containing regimens

$28(7.6)$

Other treatment

$62(16.7)$

No treatment

$10(2.7)$

$11(2.9)$

Inefficient treatment

Median antibiotic treatment duration (interquartile range)\$

Intravenous treatment $(\mathrm{N}=351)$

Total antibiotic treatment duration ( $\mathrm{N}=331)$

Aminoglycoside treatment $(\mathrm{N}=117)$

Other management

Received surgical treatment

Treatment in Intensive Care Unit (ICU)/High

dependency unit

Treatment with vasopressors

10 days (5-15)

14 days (10-25)

3 days (2-7)

119 (31.9)

$92(24.7)$

$39(10.5)$

Ventilator treatment

$15(4)$

Examined with echocardiography

134 (35.9)

Median length of stay (interquartile range)

*Data on definitive treatment lacking for two patients.

\$Data is lacking on 22 patients for intravenous treatment duration and on 42 patients for total antibiotic treatment duration.
A high proportion of our patients suffered severe sepsis or septic shock during their episode, with a striking accompanying increase in mortality even when adjusting for age, sex and comorbid burden. This strong association has been observed in several other studies [13]. The same pattern was seen for severity classified by the Pitt bacteremia score, with increased mortality for patients with a score of 2 or more. Pitt bacteremia score has been shown to be significantly correlated with mortality both in prospective clinical and ICU based trials $[30,31]$. In our cohort, nearly $70 \%$ of those with severe sepsis and $20 \%$ of those with septic shock were treated on a general hospital ward. Although care at a regular bed unit can be of very high quality and there can be various good reasons for not increasing the level of care for example a palliative setting, this still highlights the importance of well-established routines to identify and follow up these patients and ensure the best standard of care.

Assigning focus of infection of Staphylococcus aureus is challenging because of the organism's affinity to foreign objects and its propensity to generate metastatic foci [3]. We found a high mortality among patients with an unknown focus of infection. This is in line with results from previous studies $[8,32]$. The importance of ascertaining a focus of infection and removing it if possible has been well established [19]. We found lower mortality among those with bone and joint infection, abscess and primary urinary focus. The two latter can possibly be explained by the possibility to promptly remove the focus of infection in most cases. Bacteriuria accompanying Staphylococcus aureus bloodstream infection has been seen as a sign of hematogenous spread of infection with higher mortality, but ascending infection is also possible and has been linked to a more favorable outcome $[6,24,33,34]$. Surprisingly, we also found a low 30 -day mortality of endocarditis of $13.4 \%$ in our patient cohort, but this was increased by a three-fold by day 90 to $39.3 \%$. We do not have a definite explanation for this difference in short and longer term mortality, but more intensive follow-up and management in the initial phases of the disease as well as ongoing damage to valves and endocardial structures may be contributing factors. Overall, we had a low proportion of endocarditis of $4.8 \%$ in our cohort. This is lower than the $8.3 \%$ found by Kaasch et al. in a recent pooled analysis of 5 prospective observational studies [8] and much lower than a recent multicenter study in Denmark where $22 \%$ of the patients screened with echocardiography were diagnosed with endocarditis [35]. One possible explanation of the low prevalence in our cohort is underdiagnosis due to the low rate of examination with echocardiography. Rasmussen et al. identified an unknown focus of infection as one of several independent risk factors for 
Table 3 30-day mortality in relation to patient characteristics prior to infection

\begin{tabular}{|c|c|c|c|c|c|c|c|}
\hline \multirow[b]{2}{*}{ Characteristic } & \multirow[b]{2}{*}{$\begin{array}{l}\text { No. of deaths } \\
\text { within } 30 \text { days }\end{array}$} & \multirow[b]{2}{*}{$\begin{array}{l}\text { 30-day mortality } \\
\text { within category (\%) }\end{array}$} & \multicolumn{5}{|c|}{ Age- and sex-adjusted } \\
\hline & & & Odds ratio & $95 \% \mathrm{Cl}$ & $p$ & Mortality risk (\%) & $95 \% \mathrm{Cl}(\%)$ \\
\hline \multicolumn{8}{|l|}{ Age (years) } \\
\hline$<60$ & 11 & 13.6 & 1 & Reference & & 13.6 & 7.7-23.0 \\
\hline $60-69$ & 15 & 25.9 & 2.21 & $0.93-5.26$ & 0.07 & 25.9 & 16.2-38.6 \\
\hline 70-79 & 27 & 24.8 & 2.09 & $0.97-4.52$ & 0.06 & 24.8 & $17.6-33.8$ \\
\hline$\geq 80$ & 49 & 39.4 & 4.04 & $1.95-8.40$ & $<0.001$ & 38.9 & $30.8-47.8$ \\
\hline$p$ for trend & & & & & $<0.001$ & & \\
\hline \multicolumn{8}{|l|}{ Sex } \\
\hline Male & 57 & 25.3 & 1 & Reference & & 25.9 & 19.4-30.9 \\
\hline Female & 45 & 30.4 & 1.21 & $0.75-1.94$ & 0.43 & 29.5 & $21.6-36.4$ \\
\hline \multicolumn{8}{|c|}{ Charlson Comorbidity Index (CCI) } \\
\hline 0 & 11 & 12.9 & 1 & Reference & & 13.7 & $7.0-21.8$ \\
\hline $1-2$ & 41 & 25.6 & 2.13 & $1.02-4.48$ & 0.05 & 24.8 & $17.6-31.0$ \\
\hline$\geq 3$ & 50 & 39.1 & 4.29 & $2.01-9.14$ & $<0.001$ & 39.1 & $30.0-47.6$ \\
\hline $\mathrm{p}$ for trend & & & & & $<0.001$ & & \\
\hline \multicolumn{8}{|l|}{ Comorbidities\$ } \\
\hline Malignant disease & 26 & 27.1 & 0.97 & $0.57-1.66$ & 0.91 & 26.9 & $17.8-35.5$ \\
\hline Renal failure & 19 & 42.2 & 2.18 & $1.11-4.27$ & 0.02 & 41.5 & $27.1-56.3$ \\
\hline Diabetes mellitus & 21 & 29.2 & 0.99 & $0.56-1.80$ & $>0.99$ & 27.3 & $17.2-37.5$ \\
\hline Hypertension & 36 & 31.6 & 1.15 & $0.69-1.91$ & 0.6 & 29.1 & $20.3-37.3$ \\
\hline Cardiovascular disease & 51 & 33.1 & 1.29 & $0.78-2.11$ & 0.32 & 30.0 & $22.0-37.2$ \\
\hline Heart failure & 20 & 50.0 & 2.4 & $1.21-4.80$ & 0.01 & 43.8 & $28.7-59.5$ \\
\hline Chronic pulmonary disease & 25 & 40.3 & 2.01 & $1.12-3.62$ & 0.02 & 39.2 & $27.0-51.4$ \\
\hline Rheumatic disease & 15 & 38.5 & 1.84 & $0.90-3.78$ & 0.09 & 38.5 & $23.6-54.3$ \\
\hline
\end{tabular}

\$Those not having the condition in question were used as reference category for each individual comorbidity studied in this analysis.

infective endocarditis in patients with Staphylococcus aureus bacteremia, and only $29.8 \%$ of our patients with unknown focus were examined with echocardiography. It is possible that some of the patients without ascertained focus of infection had endocarditis. Underdiagnosis of endocarditis may lead to inadequate management including antibiotic treatment, and may be one factor explaining the rather high case-fatality rate in this study. The higher case-fatality rate among those with an unknown focus of infection may be due to a reluctance to carry out investigations on patients where the overall prognosis is unfavorable, such as end-of life situations. On the other hand the low performance of echocardiography and the increased case-fatality rate may also indicate that fairly simple improvements in clinical standards of assessment may help strengthen the diagnostic accuracy, treatment and hopefully outcome for this patient group.

The prevalence of MRSA in this cohort was low, and most patients received adequate antibiotic management, especially after the microbe was identified. Interestingly, nearly a third of those with community-acquired infection received inadequate therapy, often penicillin in monotherapy. This is probably a sign that the level of suspicion for this pathogen is lower than for patients that contract this infection in a healthcare-associated setting. Delay of adequate treatment is of clinical importance because of the associated increased risk of infection-related mortality [36]. There were standardized local procedures available for treating sepsis with an unknown microbe and focus, primarily recommending the use penicillin and an aminoglycoside such as gentamicin, tobramicin or netilmicin. There was no standardized guideline available for the management of Staphylococcus aureus bloodstream infection specifically, but national guidelines recommended dicloxacillin as the preferred antibiotic to treat Staphylococcus aureus infections [37]. The management of the patients and choice of treatment was at the discretion of the managing physician, as was the decision of referral to an infectious disease specialist. There was no specific local guideline recommending echocardiography for patients with Staphylococcus aureus bloodstream infections, 
Table 4 30-day mortality in relation to disease acquisition, severity, focus and time period

\begin{tabular}{|c|c|c|c|c|c|c|c|}
\hline \multirow[b]{2}{*}{ Characteristic } & \multirow[b]{2}{*}{$\begin{array}{l}\text { No. of deaths } \\
\text { within } 30 \text { days }\end{array}$} & \multirow[b]{2}{*}{$\begin{array}{l}\text { 30-day mortality } \\
\text { within category (\%) }\end{array}$} & \multicolumn{5}{|c|}{ Age-, sex- and comorbidity-adjusted } \\
\hline & & & Odds ratio & $95 \% \mathrm{Cl}$ & $\mathrm{p}$ & Mortality risk (\%) & $95 \% \mathrm{Cl}$ \\
\hline \multicolumn{8}{|l|}{ Place of acquisition } \\
\hline Community-acquired & 22 & 20.4 & 1 & Reference & & 24.2 & 14.3-31.5 \\
\hline Healthcare-associated & 48 & 30.8 & 1.18 & $0.62-2.23$ & 0.61 & 27.1 & $18.2-32.5$ \\
\hline Hospital-acquired & 32 & 29.4 & 1.42 & $0.72-2.78$ & 0.31 & 30.5 & $20.2-37.9$ \\
\hline \multicolumn{8}{|l|}{ Severity } \\
\hline Sepsis without organ failure & 27 & 12.6 & 1 & Reference & & 13.3 & 7.5-16.3 \\
\hline Severe sepsis & 45 & 40.5 & 4.97 & $2.77-8.93$ & $<0.001$ & 39.9 & $29.5-48.5$ \\
\hline Septic shock & 30 & 62.5 & 10.98 & $5.16-23.35$ & $<0.001$ & 57.3 & $42.5-72.2$ \\
\hline$p$ for trend & & & & & $<0.001$ & & \\
\hline \multicolumn{8}{|l|}{ Pitt bacteremia score } \\
\hline 0 & 26 & 20.5 & 1 & Reference & & 20.3 & $12.0-25.7$ \\
\hline 1 & 22 & 19.1 & 0.97 & $0.49-1.88$ & 0.92 & 19.8 & $11.3-25.6$ \\
\hline 2 & 21 & 32.3 & 2.30 & $1.13-4.79$ & 0.02 & 35.5 & $22.6-46.7$ \\
\hline$\geq 3$ & 33 & 50.0 & 3.66 & $1.85-7.23$ & $<0.001$ & 45.1 & $31.9-57.3$ \\
\hline $\mathrm{p}$ for trend & & & & & $<0.001$ & & \\
\hline \multicolumn{8}{|l|}{ Focus of infection } \\
\hline Unknown & 40 & 42.6 & 1 & Reference & & 38.7 & $27.5-48.2$ \\
\hline Respiratory focus & 16 & 43.2 & 1.18 & $0.52-2.70$ & 0.69 & 42.4 & $26.0-58.5$ \\
\hline Urinary tract & 4 & 12.1 & 0.18 & $0.05-0.57$ & 0.004 & 11.2 & $3.4-24.0$ \\
\hline Skin/soft tissue & 21 & 28.8 & 0.57 & $0.29-1.14$ & 0.11 & 27.6 & 16.5-37.1 \\
\hline Abscess & 3 & 11.1 & 0.26 & $0.07-1.00$ & 0.05 & 15.6 & 4.3-35.4 \\
\hline Intravenous catheter & 6 & 21.4 & 0.37 & $0.13-1.05$ & 0.06 & 20.3 & 7.8-36.3 \\
\hline Endocarditis & 2 & 11.1 & 0.22 & $0.04-1.07$ & 0.06 & 13.4 & $2.8-37.4$ \\
\hline Osteomyelitis/Septic arthritis & 5 & 11.4 & 0.23 & $0.08-0.67$ & 0.007 & 13.8 & 4.9-26.1 \\
\hline Other focus & 5 & 26.3 & 0.63 & $0.2-2.0$ & 0.43 & 29.4 & $11.4-52.2$ \\
\hline \multicolumn{8}{|l|}{ Time period } \\
\hline 1996-2003 & 35 & 24.3 & 1 & Reference & & 27.3 & $18.1-33.1$ \\
\hline 2004-2011 & 67 & 29.3 & 1.00 & $0.60-1.67$ & 0.99 & 27.4 & 19.4-31.4 \\
\hline
\end{tabular}

but the need for echocardiography in these patients was emphasized during regular internal teaching sessions when bloodstream infection or endocarditis was being reviewed.

Our study has several limitations. It is carried out in a single Norwegian region and the results are not necessarily representative for other geographic areas. We do however believe that it could be representative of the situation in many other local hospitals of similar size. The positive blood cultures were prospectively registered by our microbiology laboratory, but the clinical information was retrieved from the hospital records at a later date, which is inferior to standardized prospective clinical registration. The assignment of focus of infection was largely based on clinical assessments and investigations carried out at the time of patient admission. The investigations were carried out at the discretion of the treating clinician, and as such the diagnostic accuracy may have varied between patients. However, this data reflects the clinical everyday management and decision basis that were available for these patients. All the data were not available for all the patients, especially during the earlier part of the study and regarding antibiotic management. However, this design confers a greater level of detail and accuracy than observational studies based on discharge data.

\section{Conclusions}

Despite great efforts to improve survival of sepsis the last decade, there was no reduction in case fatality rate during the study period. There have been some promising results both of infectious disease consultation of all 
patients with Staphylococcus aureus bacteremia [38-40] and implementation of Quality of Care bundles for the management of these patients $[41,42]$. These types of initiatives along with improved measures of prevention, diagnosis and management will hopefully help improve the outcome of Staphylococcus aureus bloodstream infection in the future.

\section{Additional file}

Additional file 1: Table S1. 90-day mortality in relation to patient characteristics prior to infection. Table S2. 90-day mortality in relation to disease acquisition, severity, focus and time period. Table S3. Risk of severe sepsis/septic shock according to prior patient characteristics and infection-related characteristics.

\section{Competing interests}

The authors declare that they have no competing interests.

\section{Authors' contributions}

JP participated in design, data collection, statistical analysis, interpretation of the data and drafting of the manuscript. AM conceived the study and participated in design, data collection and drafting of the manuscript. Å participated in design, data collection and drafting of the manuscript. ES contributed to design, interpretation of the data and drafting of the final manuscript. BOA participated in design, statistical analysis, data interpretation and drafting of the manuscript. JKD participated in study design, data interpretation and drafting of the manuscript. All the authors read and approved the final manuscript.

\section{Acknowledgments}

We thank Andrew DeWan for kindly reviewing the manuscript. We thank the research nurses at Nord-Trøndelag Hospital Trust for their contribution to the data collection. We would also like to thank the staff at the Microbiology laboratory, Levanger Hospital, for consecutively including cases. This work was supported by the Liaison Committee between the Central Norway Regional Health Authority (RHA) and the Norwegian University of Science and Technology (NTNU); Nord-Trøndelag Hospital Trust's Fund for Research and Improvement; the Unit for Applied Clinical Research, Norwegian University of Science and Technology and the Norwegian Surveillance Program for Antimicrobial Resistance.

\section{Author details}

${ }^{1}$ Centre of Molecular Inflammation Research, Department of Cancer Research and Molecular Medicine, Norwegian University of Science and Technology, Trondheim, Norway. ${ }^{2}$ Department of Medicine, Levanger Hospital, Nord-Trøndelag Health Trust, Levanger, Norway. ${ }^{3}$ Department of Circulation and Medical imaging, Norwegian University of Science and Technology, Trondheim, Norway. ${ }^{4}$ Clinic of Anaesthesia and Intensive Care, St. Olavs Hospital, Trondheim University Hospital, Trondheim, Norway. ${ }^{5}$ Department of Public Health, Norwegian University of Science and Technology, Trondheim, Norway. ${ }^{6}$ Department of Infectious diseases, St Olavs Hospital, Trondheim University Hospital, Trondheim, Norway. ${ }^{7}$ Department of Endocrinology, St Olavs Hospital, Trondheim University Hospital, Trondheim, Norway. ${ }^{8}$ Faculty of Medicine, Institute of Cancer Research and Molecular Medicine, Po box 8905, N-7491 Trondheim, Norway.

\section{Received: 6 November 2014 Accepted: 19 February 2015} Published online: 04 March 2015

\section{References}

1. Laupland KB, Church DL, Mucenski M, Sutherland LR, Davies HD. Populationbased study of the epidemiology of and the risk factors for invasive Staphylococcus aureus infections. J Infect Dis. 2003;187(9):1452-9.

2. Gagliotti C, Balode A, Baquero F, Degener J, Grundmann H, Gur D, et al. Escherichia coli and Staphylococcus aureus: bad news and good news from the European Antimicrobial Resistance Surveillance Network (EARS-Net, formerly EARSS), 2002 to 2009. Euro Surveill. 2011;16(11).

3. Lowy FD. Staphylococcus aureus infections. N Engl J Med. 1998;339(8):520-32

4. Moxnes JF, de Blasio BF, Leegaard TM, Moen AE. Methicillin-resistant

Staphylococcus aureus (MRSA) is increasing in Norway: a time series analysis of reported MRSA and methicillin-sensitive S. aureus cases, 1997-2010. PLoS One. 2013;8(8):e70499.

5. Benfield T, Espersen F, Frimodt-Moller N, Jensen AG, Larsen AR, Pallesen LV, et al. Increasing incidence but decreasing in-hospital mortality of adult Staphylococcus aureus bacteraemia between 1981 and 2000. Clin Microbiol Infect. 2007;13(3):257-63.

6. Huggan PJ, Murdoch DR, Gallagher K, Chambers ST. Concomitant Staphylococcus aureus bacteriuria is associated with poor clinical outcome in adults with S. aureus bacteraemia. J Hosp Infect. 2008;69(4):345-9.

7. Mejer N, Westh $H$, Schonheyder HC, Jensen AG, Larsen AR, Skov R, et al. Stable incidence and continued improvement in short term mortality of Staphylococcus aureus bacteraemia between 1995 and 2008. BMC Infect Dis. 2012;12:260

8. Kaasch AJ, Barlow G, Edgeworth JD, Fowler Jr VG, Hellmich M, Hopkins S, et al. Staphylococcus aureus bloodstream infection: a pooled analysis of five prospective, observational studies. J Infect. 2014;68(3):242-51.

9. Asgeirsson H, Gudlaugsson O, Kristinsson KG, Heiddal S, Kristjansson M Staphylococcus aureus bacteraemia in Iceland, 1995-2008: changing incidence and mortality. Clin Microbiol Infect. 2011;17(4):513-8.

10. Mansur N, Hazzan R, Paul M, Bishara J, Leibovici L. Does sex affect 30-day mortality in Staphylococcus aureus bacteremia? Gend Med. 2012;9(6):463-70.

11. Jensen AG, Wachmann CH, Espersen F, Scheibel J, Skinhoj P, Frimodt-Moller N. Treatment and outcome of Staphylococcus aureus bacteremia: a prospective study of 278 cases. Arch Intern Med. 2002;162(1):25-32.

12. Kim SH, Park WB, Lee KD, Kang Cl, Kim HB, Oh MD, et al. Outcome of Staphylococcus aureus bacteremia in patients with eradicable foci versus noneradicable foci. Clin Infect Dis. 2003;37(6):794-9.

13. van Hal SJ, Jensen SO, Vaska VL, Espedido BA, Paterson DL, Gosbell IB. Predictors of mortality in Staphylococcus aureus Bacteremia. Clin Microbiol Rev. 2012;25(2):362-86

14. Laupland KB, Lyytikainen O, Sogaard M, Kennedy KJ, Knudsen JD, Ostergaard C, et al. The changing epidemiology of Staphylococcus aureus bloodstream infection: a multinational population-based surveillance study. Clin Microbiol Infect. 2013;19(5):465-71.

15. Levy MM, Fink MP, Marshall JC, Abraham E, Angus D, Cook D, et al. 2001 SCCM/ESICM/ACCP/ATS/SIS International Sepsis Definitions Conference. Intensive Care Med. 2003;29(4):530-8.

16. Dellinger RP, Levy MM, Rhodes A, Annane D, Gerlach H, Opal SM, et al. Surviving Sepsis Campaign: international guidelines for management of severe sepsis and septic shock, 2012. Intensive Care Med. 2013;39 (2):165-228.

17. Krokstad S, Langhammer A, Hveem K, Holmen TL, Midthjell K, Stene TR, et al. Cohort profile: the HUNT study, Norway. Int J Epidemiol. 2013;42 (4):968-77.

18. Nolte FS, Williams JM, Jerris RC, Morello JA, Leitch CD, Matushek S, et al. Multicenter clinical evaluation of a continuous monitoring blood culture system using fluorescent-sensor technology (BACTEC 9240). J Clin Microbiol. 1993;31(3):552-7.

19. Thwaites GE, Edgeworth JD, Gkrania-Klotsas E, Kirby A, Tilley R, Torok ME, et al. Clinical management of Staphylococcus aureus bacteraemia. Lancet Infect Dis. 2011;11(3):208-22.

20. Friedman ND, Kaye KS, Stout JE, McGarry SA, Trivette SL, Briggs JP, et al. Health care-associated bloodstream infections in adults: a reason to change the accepted definition of community-acquired infections. Ann Intern Med. 2002;137(10):791-7.

21. Shorr AF, Tabak YP, Killian AD, Gupta V, Liu LZ, Kollef MH. Healthcareassociated bloodstream infection: a distinct entity? Insights from a large U.S. database. Crit Care Med. 2006;34(10):2588-95.

22. Charlson ME, Pompei $P$, Ales KL, MacKenzie CR. A new method of classifying prognostic comorbidity in longitudinal studies: development and validation. J Chronic Dis. 1987;40(5):373-83.

23. Chow JW, Yu VL. Combination antibiotic therapy versus monotherapy for gram-negative bacteraemia: a commentary. Int J Antimicrob Agents. 1999;11(1):7-12.

24. Muder RR, Brennen C, Rihs JD, Wagener MM, Obman A, Stout JE, et al. Isolation of Staphylococcus aureus from the urinary tract: association of 
isolation with symptomatic urinary tract infection and subsequent staphylococcal bacteremia. Clin Infect Dis. 2006;42(1):46-50.

25. Fortin E, Rocher I, Frenette C, Tremblay C, Quach C. Healthcare-associated bloodstream infections secondary to a urinary focus: the Quebec provincial surveillance results. Infect Control Hosp Epidemiol. 2012;33(5):456-62.

26. Jacobsson G, Nasic S. Long-term outcome of invasive Staphylococcus aureus infections. Scand J Infect Dis. 2012;44(5):350-4.

27. Wyllie DH, Crook DW, Peto TE. Mortality after Staphylococcus aureus bacteraemia in two hospitals in Oxfordshire, 1997-2003: cohort study. BMJ (Clin Res Ed). 2006;333(7562):281.

28. Hanses F, Spaeth C, Ehrenstein BP, Linde HJ, Scholmerich J, Salzberger B. Risk factors associated with long-term prognosis of patients with Staphylococcus aureus bacteremia. Infection. 2010;38(6):465-70.

29. Lesens O, Methlin C, Hansmann Y, Remy V, Martinot M, Bergin C, et al. Role of comorbidity in mortality related to Staphylococcus aureus bacteremia: a prospective study using the Charlson weighted index of comorbidity. Infect Control Hosp Epidemiol. 2003;24(12):890-6.

30. Hill PC, Birch M, Chambers S, Drinkovic D, Ellis-Pegler RB, Everts R, et al. Prospective study of 424 cases of Staphylococcus aureus bacteraemia: determination of factors affecting incidence and mortality. Intern Med J. 2001;31(2):97-103.

31. Rhee JY, Kwon KT, Ki HK, Shin SY, Jung DS, Chung DR, et al. Scoring systems for prediction of mortality in patients with intensive care unit-acquired sepsis: a comparison of the Pitt bacteremia score and the Acute Physiology and Chronic Health Evaluation II scoring systems. Shock (Augusta, Ga). 2009;31(2):146-50.

32. Mylotte JM, Tayara A. Staphylococcus aureus bacteremia: predictors of 30-day mortality in a large cohort. Clin Infect Dis. 2000;31(5):1170-4.

33. Chihara S, Popovich K, Weinstein RA, Hota B. Staphylococcus aureus bacteriuria as a prognosticator for outcome of Staphylococcus aureus bacteremia: a case-control study. BMC Infect Dis. 2010;10:225.

34. Asgeirsson $\mathrm{H}$, Kristjansson M, Kristinsson KG, Gudlaugsson O. Clinical significance of Staphylococcus aureus bacteriuria in a nationwide study of adults with S. aureus bacteraemia. J Infect. 2012;64(1):41-6.

35. Rasmussen RV, Host U, Arpi M, Hassager C, Johansen HK, Korup E, et al. Prevalence of infective endocarditis in patients with Staphylococcus aureus bacteraemia: the value of screening with echocardiography. Eur J Echocardiogr. 2011;12(6):414-20.

36. Lodise TP, McKinnon PS, Swiderski L, Rybak MJ. Outcomes analysis of delayed antibiotic treatment for hospital-acquired Staphylococcus aureus bacteremia. Clin Infect Dis. 2003;36(11):1418-23.

37. Antibiotika i sykehus. IK-2737. In: Statens Helsetilsyn; 2001

38. Rieg S, Peyerl-Hoffmann G, de With K, Theilacker C, Wagner D, Hubner J, et al. Mortality of $\mathrm{S}$. aureus bacteremia and infectious diseases specialist consultation-a study of 521 patients in Germany. J Infect. 2009;59(4):232-9.

39. Fowler Jr VG, Sanders LL, Sexton DJ, Kong L, Marr KA, Gopal AK, et al. Outcome of Staphylococcus aureus bacteremia according to compliance with recommendations of infectious diseases specialists: experience with 244 patients. Clin Infect Dis. 1998;27(3):478-86.

40. Honda H, Krauss MJ, Jones JC, Olsen MA, Warren DK. The value of infectious diseases consultation in Staphylococcus aureus bacteremia. Am J Med. 2010;123(7):631-7.

41. Lopez-Cortes LE, Del Toro MD, Galvez-Acebal J, Bereciartua-Bastarrica E, Farinas MC, Sanz-Franco M, et al. Impact of an evidence-based bundle intervention in the quality-of-care management and outcome of Staphylococcus aureus bacteremia. Clin Infect Dis. 2013;57(9):1225-33.

42. Borde JP, Batin N, Rieg S, Feik R, Reimling C, Kern WV, et al. Adherence to an antibiotic stewardship bundle targeting Staphylococcus aureus blood stream infections at a 200-bed community hospital. Infection. 2014;42 (4):713-9.

\section{Submit your next manuscript to BioMed Central and take full advantage of:}

- Convenient online submission

- Thorough peer review

- No space constraints or color figure charges

- Immediate publication on acceptance

- Inclusion in PubMed, CAS, Scopus and Google Scholar

- Research which is freely available for redistribution

Submit your manuscript at www.biomedcentral.com/submit 